\title{
Design of a multi-agent system using the "MaSE" method for learners' metacognitive help
}

\author{
Hanane Elbasri, Adil Haddi, Hakim Allali \\ LAVETE Laboratory, FST, Univ Hassan $1^{\text {st }}$, Morocco
}

\begin{tabular}{l}
\hline \hline Article Info \\
\hline Article history: \\
Received Jun 24, 2018 \\
Revised Nov 19, 2018 \\
Accepted Dec 7, 2018 \\
\hline
\end{tabular}

\section{Keywords:}

AgentTool

"MaSE" method

Metacognitive agent

Multi-agent system

\begin{abstract}
This article addresses a multi-agent approach to solving the problem of integrating metacognitive incentives into Learning Management System. The behavior of the teacher in a classroom-teaching situation, where teacher adopting the competency-based approach, is characterized by a set of didactic interventions dynamically adaptable according to the actionsreactions of the learners. These interventions are continually subjected to perfection by experience. In this article, we are interested in modeling the multi-agent system in order to help the learners develop their metacognitive skills in a continuous way. The purpose of this system is to supervise the activities and statements of the learner and communicate them to the metacognitive agent. The latter focuses on the assessment of the learner's metacognitive skills in order to trigger, automatically, metacognitive incentives to provide help messages. Integrating the agent for metacognitive control and assistance, allows learners to maintain motivation and confidence, and elicit their attention to the importance of metacognitive skills during learning activity. The "MaSE" methodology and the "agentTool" are used to model the multi-agent system.
\end{abstract}

Copyright $\left({ }_{0} 2019\right.$ Institute of Advanced Engineering and Science. All rights reserved.

\section{Corresponding Author:}

Hanane Elbasri,

LAVETE Laboratory, FST,

Univ. Hassan $1^{\text {st }}$, Settat,

B.P. 577,26000 Settat, Morocco.

Email : h.elbasri@uhp.ac.ma

\section{INTRODUCTION}

Online learning offers learners the possibility to learn from a distance by freely consulting courses without a plan or guidance. Because of the absence of a teacher who supervises the learner progress by avoiding them from loosing link between the chapters, the concepts and the objectives to be reached. In other words, the teacher is precisely there to intervene, at the right moment, to respect the planning, maintain the orientation of the course, and elicit them to use the metacognitive skills. Hence, the learners need the metacognitive incentives when they learn online uniquely .

In this article, we are interested in stimulating, in distance learning, the learner to have autonomy, to be aware of the routing that leads to the goal to be attained and to notify his mistakes by himself and to seek the right solutions. This is achieved through interactions and communication between our agents that trigger the right incentives for the learner and according to his needs.

The objective of our work is to design a multi-agent system that helps the metacognitive agent to replace the teacher by collaborating with other agents (the "situation agent", the "activity agent", and the "evaluation agent"). These agents control the "perception", that is to say control the learner's situation, monitor his activity, evaluate the metacognitive skills and the degree of success. The goal is to help the metacognitive agent to distribute the appropriate incentives to the learner's needs and to further improve his metacognitive level. 
The purpose of all these interactions is to help the learner to manage his learning by means of pedagogical support, based on the learner's traces and using metacognitive help.

The metacognitive agent intervenes in the case that the learner should be in difficulty. Thanks to the agents, we actually identify the following roles:

a. motivate the passive learner in difficulty and encourage him to self-evaluate and self-regulate ;

b. encourage the learner to use metacognitive skills;

c. attract the attention of the inattentive learner;

d. collect information, manage and analyze traces.

The article is organized as follows: Section 2 describes the work related to our study. Section 3 describes the methodological approach and, in Section 4, we present the modeling of our multi-agent using the "MaSE" methodology. Finally, in Section 5, we present our conclusions and perspectives.

\section{RELATED WORKS}

Metacognition can take many forms. It includes knowledge about when and how to use learning strategies [1]. Encouraging learners to think metacognitively should have a positive impact on learning performance regardless of the form of learning [2], [3]. Some works have focused on metacognitive integration initiated by the teacher, from time to time, during face-to-face learning. These studies show that this kind of metacognitive intervention helps the learner as Liliane Portelance does in 2002 [4].

In 2009, a relationship was found, by IzaskunIbabe and Jauregizar [5], between self-evaluation, on the one hand, and learner effort and performance, on the other. In 2012, the effect of computer-assisted learning with metacognitive incentives on students' actual skills was the focus of the work of Nilgün and his team [6]. In 2013, support for student self-regulation and learning with an open learner model with a linear equation tutor was presented by Long and Aleven [7]. In 2014, Donna Bryce and her team investigated the relationships between executive functions, metacognitive skills, and academic achievement in children aged 5 and $7[8]$.

In class and on-line learning, some researches have been done to identify tools for improving metacognitive skills in learners, as is the case with Bernard's team in 2015 [9]. Other works discuss the characteristics of a metacognitive support system. This is particularly the case of the work of MohdRum and others in 2017 [10]. Other studies have focused their research on improving platforms in distance education to help the learner to follow their studies. The problem with these platforms is that, in all the works, the focus has been on the integration of agents at the cognitive level of the learner even if we observe the abandonment of the continuation of learning.

In 2017, a relationship was found between learning styles and learner behavior in online learning by Baharudin and his teammates [11]. Juliane and her team said that motivation is undeniable for the success of e-learning processes [12]. The previously cited review of literature shows the impact of a metacognitive skill on the learners' performance or the success of the training. However, we didn't find the work has addressed the relationship between metacognitive skills and learner status and does not introduce adequate incentives according to the learner's state of mind. In the following section, we focus on the link between the learner's state and the metacognitive incitement associated with it.

Multi-agent systems (MAS) require interactions between agents. These interactions lead to the achievement of the common goal. Scott and Wooldridge used agent-oriented methodologies such as MaSe [13], [14] GAIA [15] and other suitable modeling techniques. It has been found that some projects use multi-agent systems to develop their approach in the learning domain as Baghera architecture [16], ALLEGRO environment [17] etc.

The "MaSE" methodology is a seven-step, and a two-phase process. In the analysis phase, it begins with the structuring of the objectives, the identification of the use cases and the definition of the roles. Whereas, in the design phase, it defines the agent diagram, the sequence diagram, the communication or conversation diagram between classes, and the deployment diagram [13], [14]. In 2007, K. Harbouche and M.Djoudi [18] used the "MaSE" methodology to model an agent-based virtual assistant for cognitive distance education.

\section{METHODOLOGICAL APPROACH}

Our starting point was a test that we conducted at the Faculty of Science and Technology of Settat (Morocco) in July 2017 with students in a computer class. In this test, we introduced metacognitive incentives into our learning system. The results show that students - who benefited from these metacognitive incentives - had a success rate of $71 \%$ compared to only $30 \%$ for students who did not benefit. In the Table 1 , 
we show the association between the states of the learners and the actions to be performed by the metacognitive agent, a table resulting from our work done in 2017 [19].

Table 1. Association between States and Actions to be Performed by the Metacognitive Agent [19]

\begin{tabular}{ll}
\hline \multicolumn{1}{c}{ State } & \multicolumn{1}{c}{ Incentives related to the state of the agent } \\
\hline Disoriented & Encourage the planification \\
Confused & Encourage the use of self-assessment strategies and self-regulation strategies \\
Improving & Encourage the use of metacognitive strategies and the links between concepts \\
& and teaching units \\
Failure & Encourage the learner to plan, self-regulate and memorize strategies \\
\hline
\end{tabular}

The associations lead us to suggest, first and foremost, the identification of the learner's statement by interactions between the different agents used (see Table 2), that is to say the communication of our agents are as follows:

a. The Activity_Agent sends to the Situation_Agent the activity of the learner (active, passive) ;

b. The Situation_Agent communicates to the Metacognitive_Agent the situation of the learner (absent, in difficulty, fast, slow);

c. The Metacognitive_Agent then triggers the metacognitive incentives by targeting them according to the state of the learner during the learning process;

d. The Evaluation_Agent evaluates and sends the level of the learner to the Metacognitive_Agent.

Table 2. Typology of the Agents used

\begin{tabular}{ll}
\hline \multicolumn{1}{c}{ Agent } & \multicolumn{1}{c}{ Comment } \\
\hline $\begin{array}{l}\text { Activity_Agent } \\
\text { Situation_Agent }\end{array}$ & These are "reactive agents", agents that use the "stimulus-reaction", type of capability \\
$\begin{array}{l}\text { Evaluation_Agent } \\
\text { Metacognitif_Agent }\end{array}$ & $\begin{array}{l}\text { It has a specific goal to achieve with a well-developed action plan. This agent is able to assist } \\
\text { the learner when needed by communicating with other agents [20], [21]. }\end{array}$ \\
\hline
\end{tabular}

As a conclusion: the metacognitive agent focuses first on the state of the learner, follows it state by state, triggers the appropriate incentives (Table 1) and thus, gradually and implicitly, inculcates metacognitive skills.

\section{MODELING THE SYSTEM}

The issue dealt with, in this article, is to treat the interactions between our agents to detect the state of the learner and to transmit it to the metacognitive agent. When the learner is in difficulty, the Metacognitive_Agent receives his "state" from the Situation _Agent and reacts in order to assist him to overcome his difficulties. In order to retrieve the learner's state, the agents ask the learner questions and analyze the answers which are provided by agents in ordre to determine the learner status. In fact, the metacognitive agent can assist the learner before, during and after the course consultation.

In this part, a multi-agent system can be defined as a set of autonomous unites, called "agents" that interact and communicate with each other through well-defined rules in a specific environment to achieve specific goals. Several methodologies allow to model and develop multi-agent systems. Our choice was on the Organization Multi-agent System engineering methodology (O-MaSe) [22], [23] - which is an extension of "MaSE" - to model our system. It allows, in all freedom, to move between the different phases of the system and to proceed, dynamically, to management [24]. This simple method is easy to use and is validated by the "agentTool". In the next section, we present the different diagrams, namely the goal and role diagram, the agent diagram and the protocol diagrams..

\subsection{Goal diagram}

The main purpose of this diagram is the "learner metacognitive incitement" which depends on the evaluation of metacognitive competence [goal (1.1)], the monitoring of the learner's activities [goal (1.2)], and the assessment of the degree of understanding [goal (1.3)]. Incentives in the form of messages will be triggered, at any given moment, to retrieve information about planning, motivation, strategy, trust, selfregulation, and learner self-assessment [goal (1.1)].

Goal (1.2): to collect the necessary data on the speed of the reading of the courses, to treat the requests of the learner (to detect the difficult words ....), and the taking of the notes. Goal (1.3) of the diagram 
presents tests predefined by the teacher at the time of deposit of the courses in a learning environment. These tests are composed in two sub-goals. In fact, the new components help to follow the profile of the learner, that is to say that the level of the learner's understanding is taken into consideration goal (1.3.1). The goal (1.3.2), we take into account as information the follow-up of the memorizing capacity of the learner. In Figure 1 we can see the hierarchy of goals.

\subsection{System role diagram}

In this part, we present the different roles played by our system and the capabilities it provides. Whereas, in the "O-MaSE" methodology, we consider the learner as an external resource, the roles - in the model - show the interactions between the role tasks. Roles are indicated by rectangles, while oval shapes indicate tasks. The arrows, between tasks, indicate the protocols. This diagram describes the different roles that can be played by system agents to achieve previously identified goals or subgoals. From these goals, we can identify the roles on Table 3. The MaSE role model can we see in Figure 2.

\subsection{The system agents}

This diagram makes it possible to visualize the MAS and its interactions between the agents. These later need to communicate with learners to make the right decisions. The relations between the classes of the agents indicate the relation between "initiator" and "responder" (arrow pointed from the "initiator" side to the "responder", the line indicating the messages which circulate between them). In the following paragraphs, we present the conversation that defines the coordination protocol between two agents (A_MET, A_SIT) using two communication diagrams (initiator, responder) as shown in Figure 3.

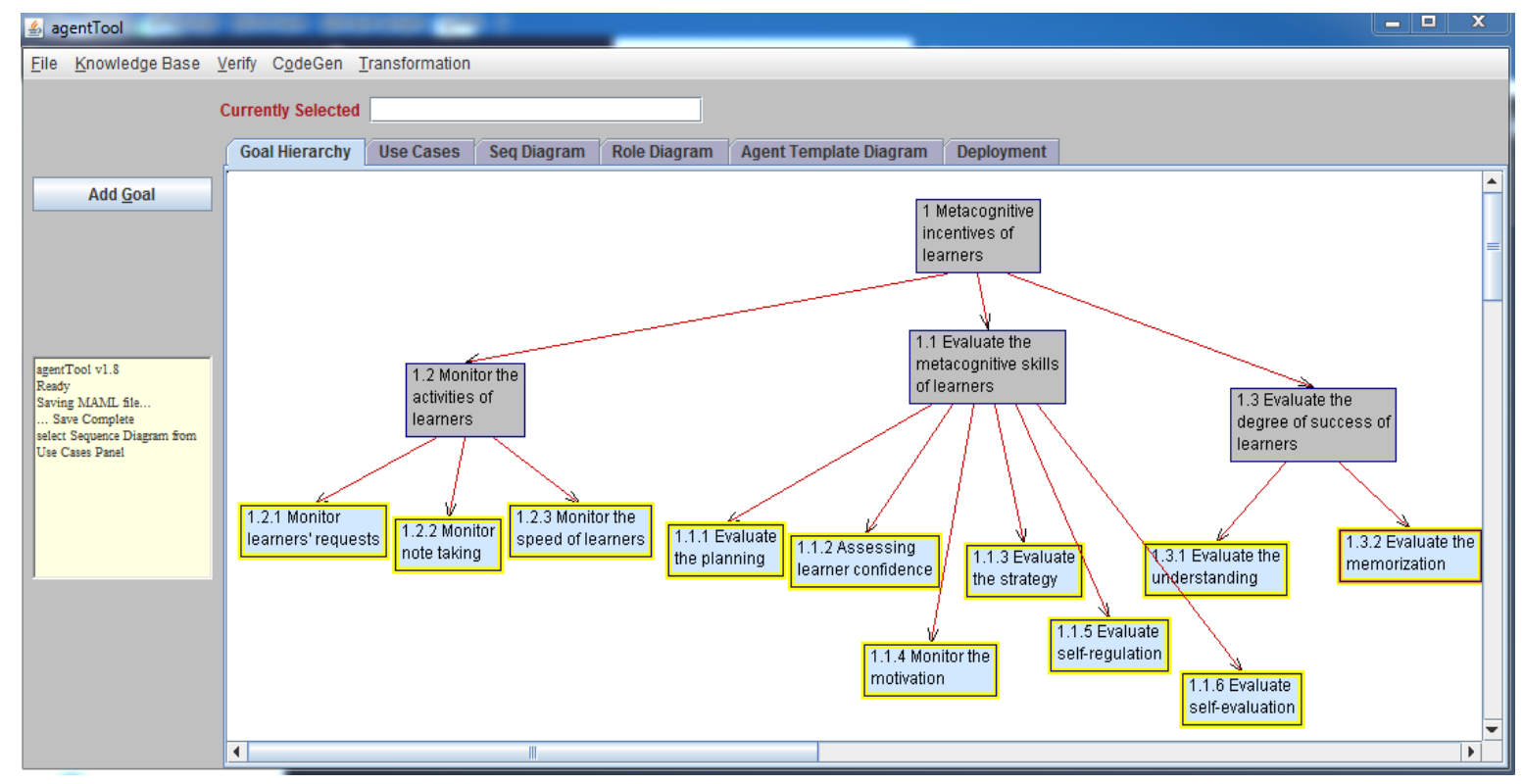

Figure 1. Hierarchy of goals

Table 3. Roles and Capabilities

\begin{tabular}{ll}
\hline \multicolumn{1}{c}{ Roles } & \multicolumn{1}{c}{ Capabilities } \\
\hline To control the activity & $\begin{array}{l}\text { Responsible for collecting the following data: entering the consultation time, } \\
\text { recording the learner's comments, detecting the learner's state (active, passive) } \\
\text { Control or assist the learner with motivation, caution, and trust during reading and } \\
\text { send the learner's state to the incentive agent to play his role } \\
\text { Responsible for knowing whether the learner is "planned" or not and using the } \\
\text { incentive agent to encourage the learner to organize his work } \\
\text { Re control the organization } \\
\text { To control learning }\end{array}$ \\
$\begin{array}{l}\text { To control the metacognitive } \\
\text { skills }\end{array}$ & \begin{tabular}{l} 
Helps to support the learner through self-assessment, strategy testing \\
\hline
\end{tabular}
\end{tabular}




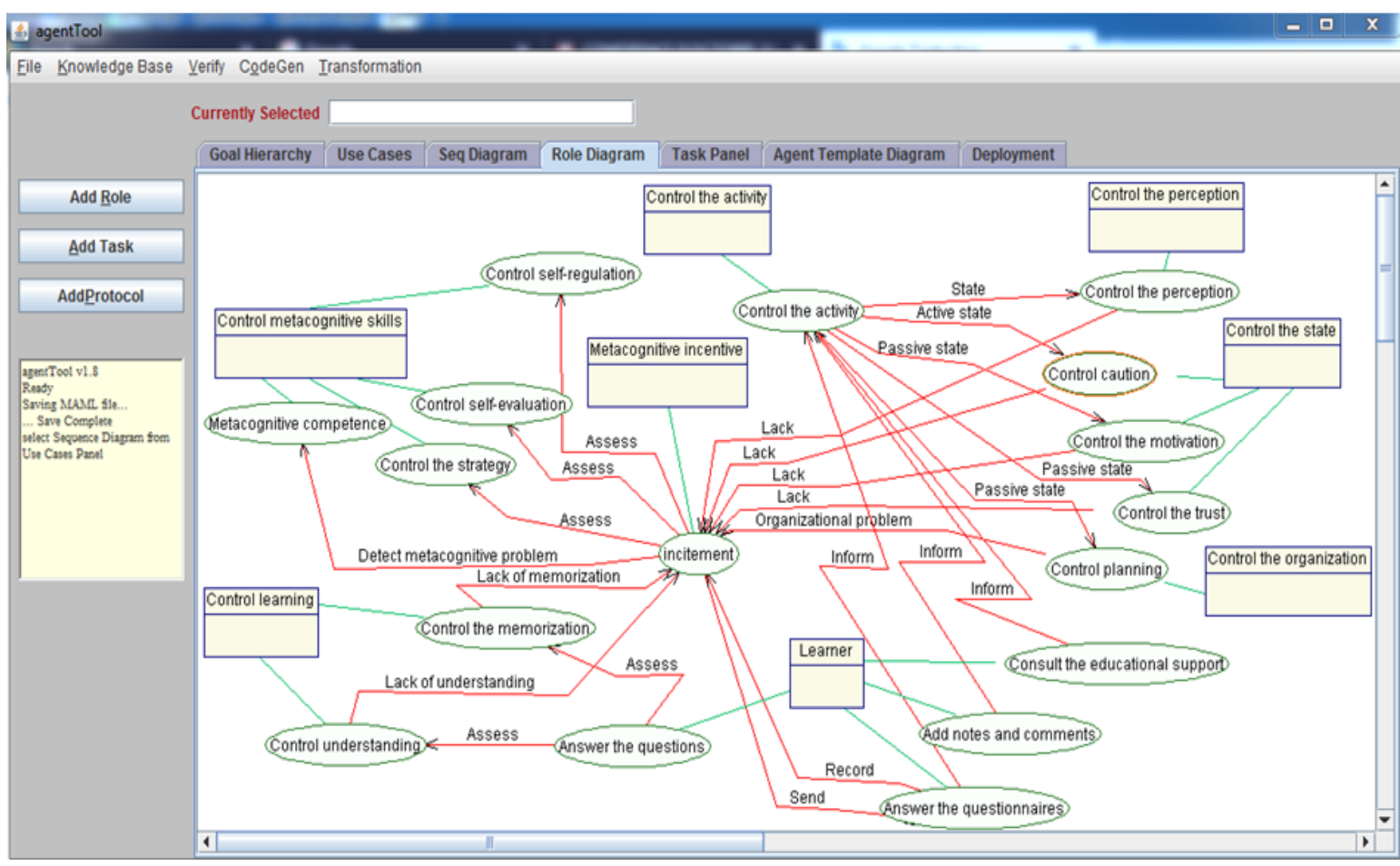

Figure 2. MaSE role model

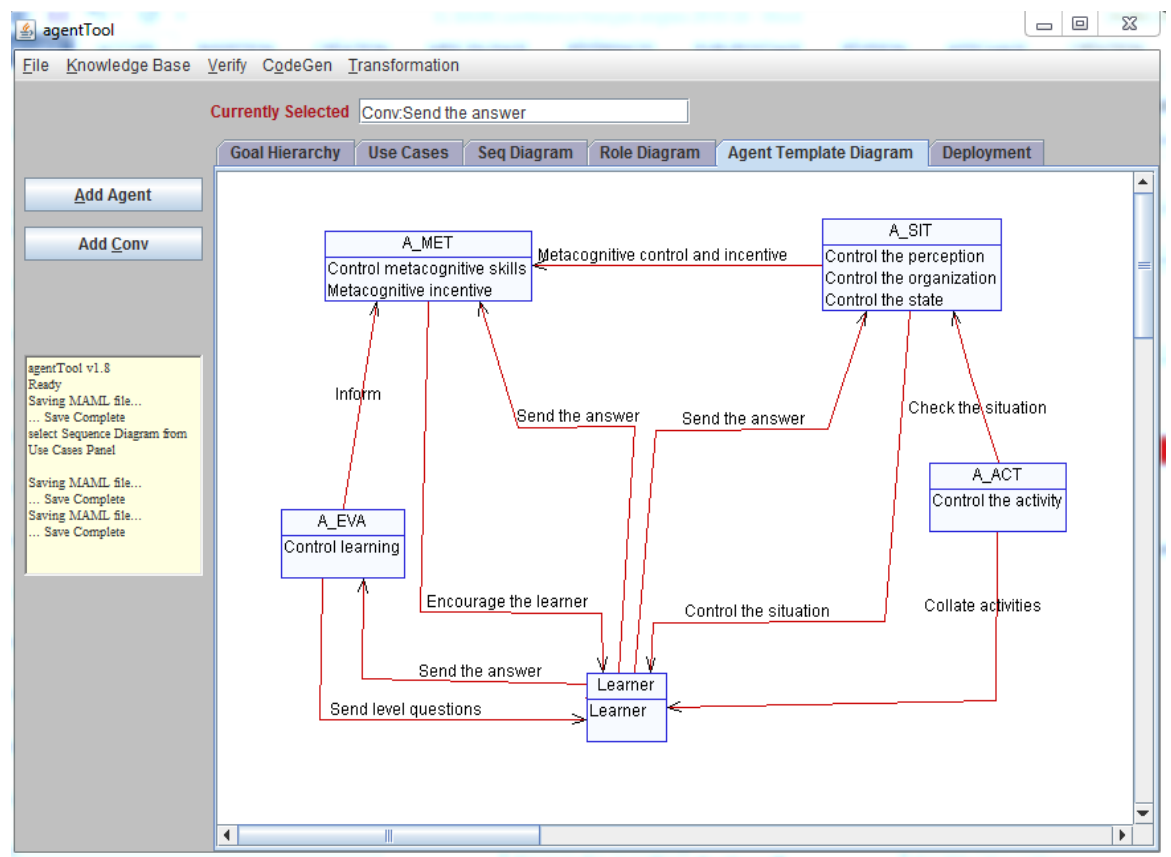

A_ACT: Activity_Agent A_STI: Situation_Agent A_EVA: Eraluation_Agent A_MET:Metacognitif_Agent

Figure 3. Agent class diagram

\subsection{Metacognitive control and incentives (initiator and responder)}

Once the agents are created, we develop their behaviors. An agent can have multiple tasks. Each task is specified as a state machine, and is composed of states and transitions. The states represent the processes (activities) performed by the agent and a transition consists of a source-state and one or more destination states, a trigger, a guard condition, and a transmission. Transition uses the following syntax:

\section{Trigger [Guard] ${ }^{\wedge}$ Transmission}


Two special events, "send" and "receive", are used to indicate messages to be exchanged between agents. The "send" event is used to send a message to another agent, the syntax of this event is: send (messge, agent). The "receive" event is used to indicate the receipt of a message from another agent, the syntax of this event is: receive (message, agent).

A message is described by the intent of message and is associated with a set of parameters. The states contain activities that represent internal reasoning, the reading of perception from sensors. In Figure 4, the conversation begins when the agent "A_SIT" receives a passive state of the learner. This event causes, then, a transition of the agent "A_SIT" from the waiting state to the state of control of the situation of the learner. Depending on the result of the learner status check - which can be positive (absent) or negative (in difficulty) - the agent "A_SIT" goes to the waiting state or displays a dialog box containing questions about the difficulties encountered and goes to the waiting state of response and that respectively. After evaluating the learner's response, the agent "A_SIT" goes to the waiting state if the situation is good, otherwise it sends the type of difficulty to the agent "A_MET".

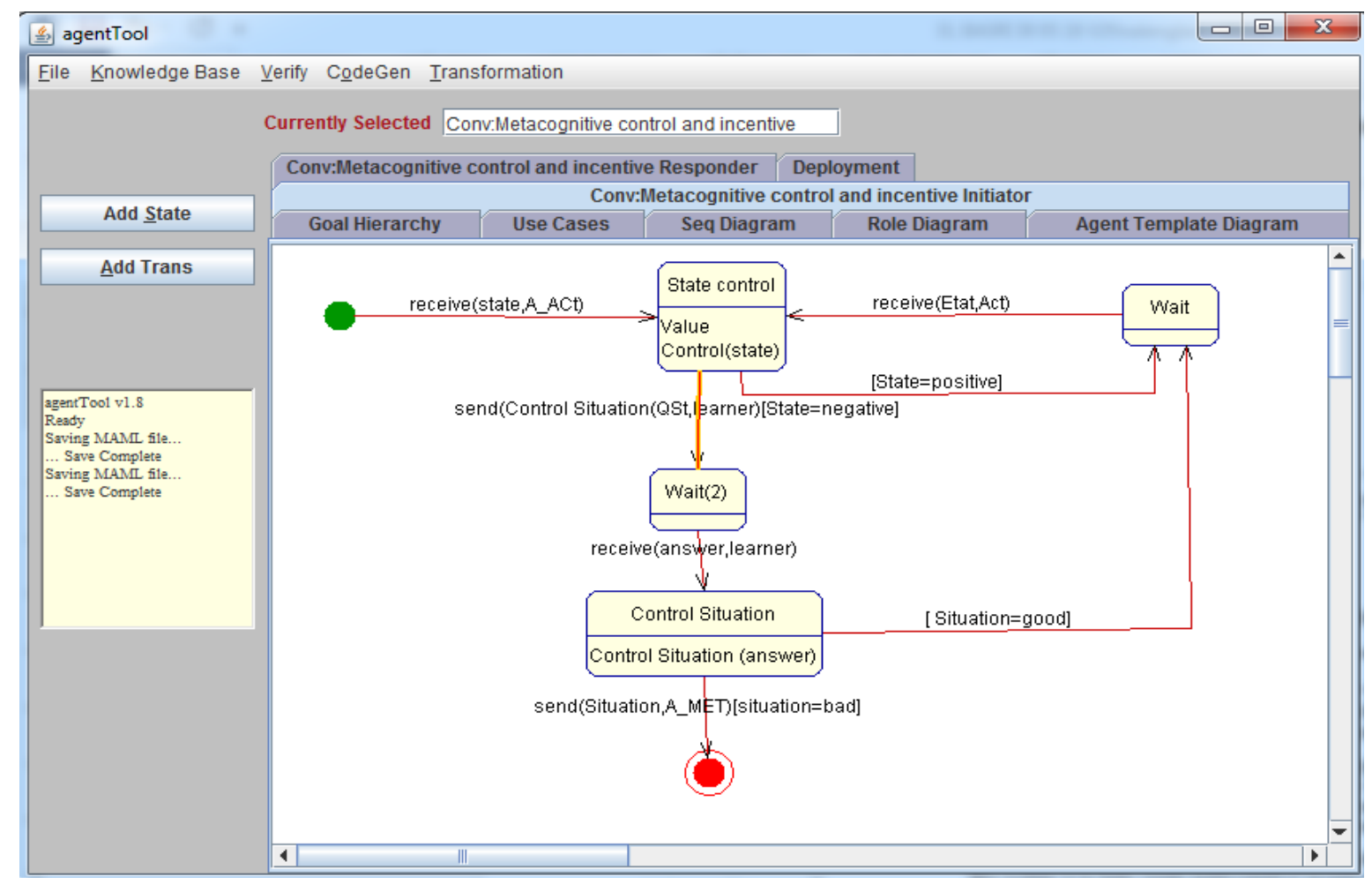

Figure 4. Control of the diagram conversation situation for A_SIT agent

In Figure 5, the conversation begins when the agent "A_MET" receives the situation of the learner from the agent "A_SIT". This event causes the transition of the agent "A_MET" from the waiting state to the state of control of the learner situation and this according to the result of the activity of control of the situation which can be good or bad. In the case where the situation is good, the agent "A_MET" goes to the waiting state. On the other hand, in the case where the situation is bad, the agent sends a questionnaire to check the metacognitive level of the learner and goes to the waiting state of response. Once the agent receives the response from the learner, he evaluates the answer : if the learner has a good metacognitive level, the agent goes to the waiting state and, at the same time, sends a message of congratulations to the learner, otherwise he sends him a metacognitive insight. 


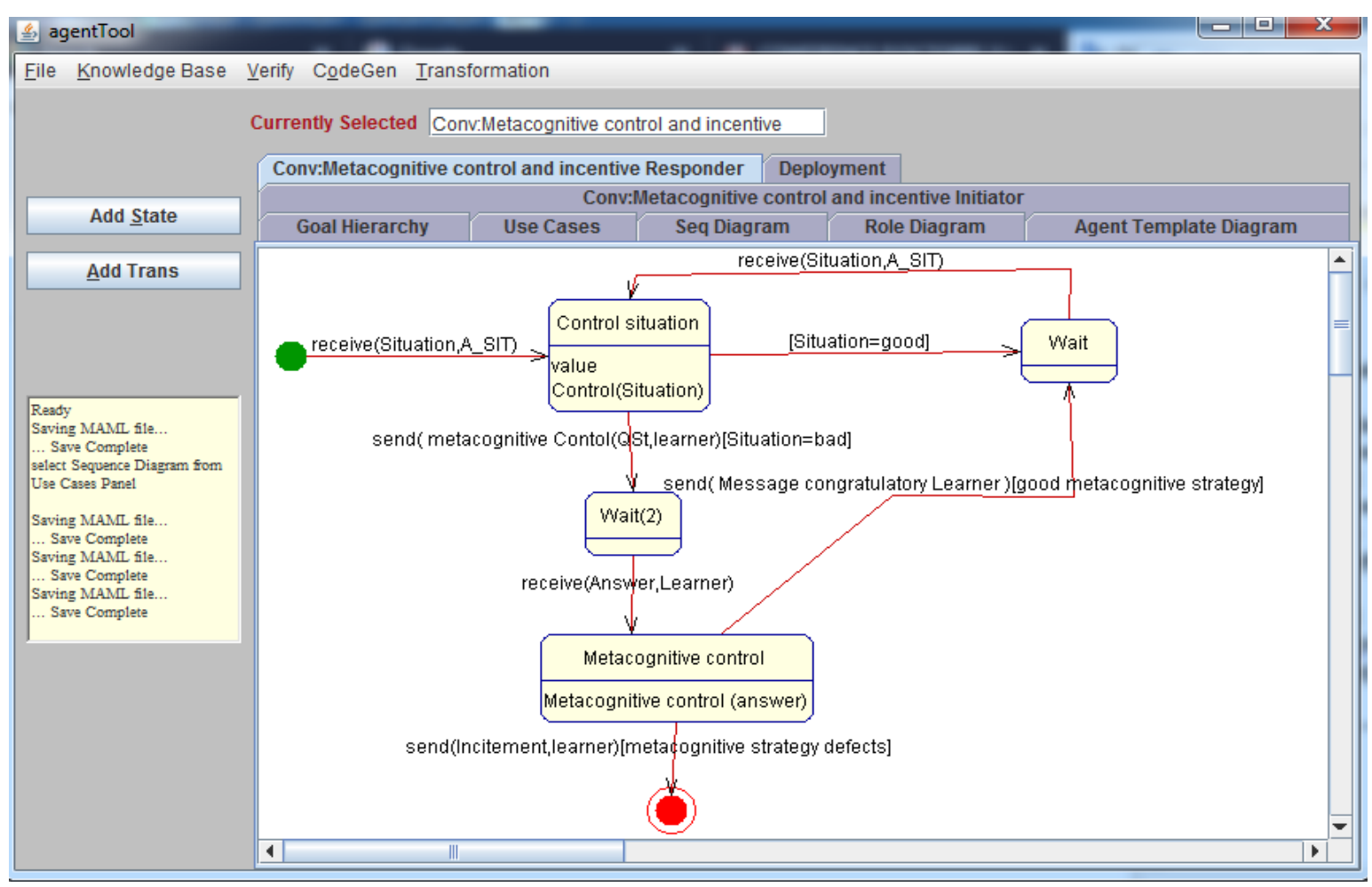

Figure 5. Incitation conversation diagram for A_MET agent

\section{CONCLUSION AND PERSPECTIVES}

The article presents a conception of a multi-agent system for the learners metacognitive incentive. The modeling of the system was done according to the "MaSe" methodology. The different diagrams have been developed and validated using the "agentTool" tool. This system is composed of several agents who collaborate to develop the autonomy and metacognitive skills of learners. In a future work, we intend to build a multi-agent system model that can communicate with the LMSs (Learning Management System) and dynamically extract the metacognitive agent action plan.

\section{REFERENCES}

[1] Metcalfe J. and Shimamura A., "Metacognition: Knowing About Knowing,” Bradford Books, Cambridge, 1994.

[2] T. Lehmann, et al., "Cognitive, metacognitive and motivational perspectives on preflection in self-regulated online learning," Computers in Human Behavior, vol. 32, pp. 313-323, 2014.

[3] Mehrak R. and Maral K., "The role of metacognitive listening strategies a wareness and podcast-use readiness in using podcasting for learning English as a foreign language," Computers in Human Behavior, vol. 28, pp. 1153$1161,2012$.

[4] L. Portelance, "Intégrer la métacognition dans lensemble de ses interventions pédagogiques," vol. 122, pp. 20-23, 2002.

[5] Jauregizar and I. I. Æ. Joana, “Online self-assessment with feedback and metacognitive knowledge,” High Educ, Springer Science+Business Media, pp. 243-258, 2010.

[6] Nilgün T., et al., "The Effect of Computer-Assisted Learning Integrated with Metacognitive Prompts on Students' Affective Skills," J Sci Educ Technol, Springer ScienceBusiness Media. New, 2012.

[7] Long Y. and Aleven V., "Supporting Students' Self-Regulated Learn with an Open Learner Model in a Linear Equation Tutor,” AIED, LNAI 7926, Springer-Verlag. Berlin Heidelberg, pp. 219-228, 2013.

[8] Bryce D., et al., "The relationships among executive functions, metacognitive skills and educational achievement in 5 and 7 year-old children," Metacognition Learning, Springer Science+Business, pp. 181-198, 2014.

[9] M. Bernard and E. Bachu, "Enhancing the metacognitive skill of novice programmers through collaborative learning," Metacognition: Fundaments, applications, and trends, pp. 277-298, 2015.

[10] S. N. M. Rum and M. A. Ismail, "Metocognitive Support Accelerates Computer Assisted Learning for Novice Programmers," Educational Technology \& Society, vol/issue: 20(3), pp. 170-181, 2017.

[11] Baharudin A. F., et al., "Behavioral tracking in E-learning by using Learning styles approach," IJEECS, vol/issue: 8(1), pp.17-26, 2017.

[12] Juliane C., et al., "Predicting the presence of learning Motivation in Electronic learning: A New Rules to Predict," TELKOMNIKA (Telecommunication Computing Electronics and Control), vol/issue: 15(3), pp 1223-1229, 2017.

Design of a multi-agent system using the "MaSE" method for learners' metacognitive help (Hanane Elbasri) 
[13] S. A. Deloach, et al., "Multiagent systems engineering," International Journal of Software Engineering and Knowledge Engineering, vol/issue: 11(3), pp. 231-25, 2001.

[14] S. A. DeLoach, "Analysis and Design using MaSE and agentTool," 12th Midwest Artificial Intelligence and Cognitive Science Conference (MAICS 2001)Miami University, Oxford, Ohio, 2001.

[15] Wooldridge M., et al., "The Gaia Methodology for Agent-Oriented Analysis and Design," Journal of Autonomous Agents and Multi-Agent Systems, vol/issue: 3(3), pp. 285-312, 2000.

[16] S. Pesty, et al., "Baghera:une architecture multi-agents pour l'apprentissage humain," Cognitique, in P. Aniorte and S. Gouardereseds, Cepadeus Edition, Toulouse, 2003.

[17] R. M. Viccari, et al., "ALLEGRO: Teaching/Learning Multi-Agent Environment using Instructional Planning and Cases- Based Reasoning (CBR)," CLEI Electronic Journal, vol/issue: 10(1), 2007.

[18] K. Harbouche and M. Djoudi, "Assistant virtuel à base d'agents pour un enseignement à distance," 4th International Conference on Computer Integrated Manufacturing CIP'2007, 2007.

[19] H. Elbasri, et al., "Improving E-learning by Integrating a Metacognitive Agent," International Journal of Electrical and Computer Engineering (IJECE), 2018.

[20] Hamid B., et al., "Architectural Approaches for Self-Healing Systems Based on Multi Agent Technologies," International Journal of Electrical and Computer Engineering (IJECE), vol/issue: 3(6), pp. 779-783, 2013.

[21] Lotfi E., et al., "Towards a System of Guidance, Assistance and LearningAnalytics Based on Multi- Agent System Applied on SeriousGames," International Journal of Electrical and Computer Engineering (IJECE), vol/issue: 5(2), pp. 344-354, 2015.

[22] Deloach S. A., "Engineering Organizationbased Multiagent Systems," 4th International Workshop on Software Engineering for Largescale multiagent Systems (SELMAS'05), vol. 3914, pp. 109-125, 2005.

[23] Deloach S. A., "Multiagent Systems Engineering : A Methodology And Language for Designing Agent Systems," 1999.

[24] S. A. DeLoach and W. H. Oyenan, "An Organizational Model and Dynamic Goal Model for Autonomous, Adaptive Systems,” Technical Report No. MACR-TR-2006-01, Kansas State University, 2006.

\section{BIOGRAPHIES OF AUTHORS}
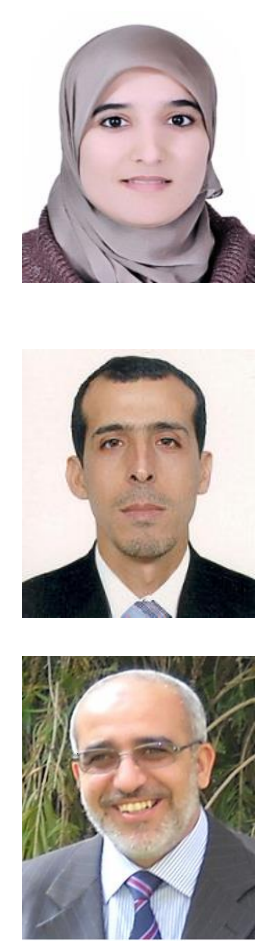

Hanane Elbasri is currently in charge of the IT department at the Beni-Mellal regional court of auditors, Morocco, North Africa. She received her Master degree in biomedical genius (instrumentation and maintenance) from Settat University, Morocco, in 2013. Online learning and, in particular, the modeling of metacognitive aspects, is her main focus.

Adil Haddi is currently computer science professor at Berrechid High Technology School, Morocco, North Africa. He received his Ph.D. degree in computer science from Hassan II University, Casablanca, Morocco, in 2009. Its research centers focus on software, datamining, e-commerce and e-learning.

Hakim Allali is currently professor of computer science at Faculty of Sciences and Technologies of Hassan 1st University of Settat in Morocco and director of LAVETE (watch laboratory of emerging technologies). He is executive manager and founder of Settat IT Learning Campus. He received his Ph.D degree in nuclear instrumentation from Claude Bernard Lyon I University, France, in 1993 and the "docteur d'Etat" degree in surface analysis, modeling and simulation from Hassan II University, Casablanca, Morocco, in 1997. He is particularly interested in technology enhanced learning, modeling, image processing and computer networking. 